Cultures \& Conflits

54 | été 2004

Approches critiques de la sécurité

\title{
Production des savoirs et représentations stratégiques
}

Christophe Wasinski

\section{OpenEdition}

1 Journals

\section{Édition électronique}

URL : http://journals.openedition.org/conflits/1549

DOI : 10.4000/conflits. 1549

ISSN : $1777-5345$

Éditeur :

CCLS - Centre d'études sur les conflits lilberté et sécurité, L'Harmattan

\section{Édition imprimée}

Date de publication : 1 juin 2004

Pagination : 163-180

ISBN : 2-7475-7301-X

ISSN : 1157-996X

\section{Référence électronique}

Christophe Wasinski, "Production des savoirs et représentations stratégiques », Cultures \& Conflits [En ligne], 54 | été 2004, mis en ligne le 08 janvier 2010, consulté le 30 mars 2021. URL : http://

journals.openedition.org/conflits/1549; DOI : https://doi.org/10.4000/conflits.1549

Ce document a été généré automatiquement le 30 mars 2021.

Creative Commons License 


\title{
Production des savoirs et représentations stratégiques
}

\author{
Christophe Wasinski
}

1 Depuis la fin de la Guerre Froide environ, les études de sécurité sont le théâtre d'un important débat relatif à la définition de leur champ intellectuel. Pour certains chercheurs, le terme de sécurité devait être reconceptualisé dans un sens plus large et plus conforme à de nouvelles pratiques. Une telle démarche va de paire avec l'usage quasi immodéré du concept de sécurité (sécurité alimentaire, sécurité économique, sécurité informatique, sécurité sociale, etc.) par les acteurs sociaux internationaux et internes ${ }^{1}$.

2 La tendance actuelle (surtout en Europe ?) est donc de réduire la visibilité des aspects les plus traditionnels du domaine de la sécurité, c'est-à-dire les questionnements relatifs à la guerre et à la stratégie dans leurs dimensions les plus militarisées. Positionnement parfois paradoxal si l'on en juge par l'affirmation, de la part de nombreux chercheurs, que ces deux phénomènes constituent toujours une dimension essentielle, si ce n'est l'épicentre, des études de sécurité2.

3 On ne peut douter, selon nous, que les débats contemporains sur l'extension de la sécurité s'avèrent non seulement riches mais également fondamentaux à la compréhension de nos sociétés. Néanmoins, on ne peut non plus négliger les apports actuels portant sur la guerre et la stratégie militaire. D'une part, et malheureusement, la guerre n'a pas disparu du paysage international. D'autre part, de par la virulence de leur expression, guerre et stratégie permettent souvent de jeter un regard décalé sur les autres formes de conflictualités.

Dans ce contexte, il convient de mettre en évidence quelques travaux, de façon non exhaustive, consacrés à ces thématiques. Ces travaux ont été choisis car ils avaient pour caractéristique commune de ne pas s'appliquer à un "monde préétabli» mais de soulever le problème de la "création de la réalité ». Ils ont donc, entre autres, pour objet d'étude l'élaboration de représentations, le développement des savoirs et les effets de ceux-ci. Dans un second temps, nombre de ces analyses ont pour objectif de montrer comment ces constructions sont liées à des relations de pouvoir, comment 
elles servent à réifier ces relations, comment certains agents s'en servent pour produire et reproduire un ordre social. Enfin, elles ont pour point commun, suite à l'effort de déconstruction entrepris, de faciliter le travail d'imagination pour la création d'un monde différent ${ }^{3}$.

5 En ce sens, ces travaux contribuent à une démarche intellectuelle constructiviste à condition d'entendre le terme dans un sens large ${ }^{4}$ et non pas, dans la plupart des cas, le rattacher au sens plus précis que lui donne le constructivisme en Relations Internationales actuellement ${ }^{5}$.

6 L'intégration entre ces travaux, provenant en grande partie de l'histoire et du constructivisme en Relations Internationales n'est donc pas, à proprement parler, réalisée. Néanmoins, ils apportent un éclairage particulier quant aux problèmes de politique internationale.

7 Tout d'abord, au travers de certains d'entre eux, le fait que le champ de bataille, ou la zone de combat, soit rarement appréhendé par les politologues en Relations Internationales comme un espace légitime de son savoir est « souligné en creux ». Or, cet espace est bel et bien un espace de lutte politique et, régulièrement, international. Par ce biais, il s'agirait de mieux prendre en compte les effets de la politique internationale sur des groupes de " petites » tailles (et inversement).

8 Ensuite, car la production de savoirs stratégiques utilisés et éventuellement créés par les politologues peut aussi être contextualisée au sein d'une démarche critique (où l'on exposera les liens entre discours théorique et position institutionnelle par exemple).

De façon générale, les travaux présentés dans les lignes qui suivent ont ceci en commun d'aborder, au travers d'approches diverses, une question centrale de la démarche constructiviste (et critique) en Relations Internationales: celle de la création de l'Autre, de son identité et des effets qui en découlent. Ici, on verra comment divers auteurs ont abordé ce problème et l'ont mis en relation avec la question du déni d'humanité, de la brutalisation des conflits, de la défense d'intérêts institutionnels et étatiques ou de la fermeture du monde.

\section{Histoire et sociologie militaire}

10 La réflexion sur l'activité guerrière s'est traditionnellement traduite par un intérêt marqué pour tout ce qui relève du combat. Tout aussi traditionnellement, à partir de l'Antiquité, le combat a longtemps été étudié par la pensée stratégique classique (Végèce, Machiavel, Jomini, Clausewitz, etc.) et l'histoire militaire dite "histoire bataille ». Bien qu'une lecture attentive des textes classiques en la matière révèle un intérêt réel, mais dispersé, pour la place de l'individu sur le champ de bataille, la perspective de cette littérature est en majeure partie celle du chef de guerre manipulant « froidement » des unités dans l'espace.

11 C'est principalement avec la Seconde Guerre mondiale qu'une réflexion psychosociologique se développe, plaçant le soldat individuel ou par petits groupes au cœur de l'analyse. Celle-ci tente d'expliquer pourquoi et comment le soldat combat. Le principal élément mis en évidence est le rôle de la cohésion sociale au sein des groupes primaires et la force de la pression sociale qui oblige les hommes à faire feu et à risquer leur vie ${ }^{6}$. 
12 Ces recherches psychosociologiques sur le combat, dont un nombre important est sponsorisé par le Pentagone, vont connaître un développement non négligeable pendant les premières décennies de la Guerre Froide. Toutefois, elles sont peu fertilisées par un croisement avec l'histoire militaire. Sous l'influence de l'école des Annales, l'histoire militaire a tendance à s'effacer jusqu'au cours des années 1970. Elle connaît ensuite un retour en force au travers de la «nouvelle histoire militaire » et de la «nouvelle histoire bataille $»^{7}$ dont l'arrivée est bien symbolisée par l'apparition de l'ouvrage The Face of Battle de John Keegan en $1976^{8}$. Dans ce best-seller de l'histoire militaire, l'auteur " humanise " l'étude du phénomène en mettant en évidence le rôle de l'individu dans la bataille9. La voie " humaniste » ouverte produit deux catégories de travaux, catégories non exclusives. D'une part, des travaux intéressés par les conditions de vie et les représentations pendant les conflits (aussi bien pour les civils que pour les militaires) voient le jour. Ceux-ci cherchent à rendre compte de la réalité vécue par les individus en temps de guerre en étudiant des phénomènes aussi divers que la consommation d'alcool, les privations, ou les stéréotypes ${ }^{10}$. D'autre part, des travaux plus directement consacrés aux activités violentes, et donc au combat, commencent à paraître. Parmi ces derniers, les analyses d'Omer Bartov, de Craig M. Cameron et de Michael Doubler méritent en particulier d'être mentionnées.

\section{Combats et dénis d'humanité}

L'étude d'Omer Bartov porte sur la "brutalisation » (barba-risation) du comportement des hommes de l'armée allemande sur le front de l'Est pendant la Seconde Guerre mondiale ${ }^{11}$. L'un des intérêts de son étude est d'analyser ces comportements non pas au sein des unités SS mais dans la Wehrmacht, souvent dédouanée des atrocités. Bartov montre comment des facteurs de type idéologique (nazisme) ont pu jouer un rôle important en influençant la perception que les soldats avaient de leur environnement. Plus précisément, ces facteurs ont participé à la négation de l'humanité de l'adversaire et favorisé les exactions. A cela s'ajoute une sorte de jeu de miroirs. Imputant des caractéristiques inhumaines, bestiales, à l'ennemi, les Allemands finirent par devenir eux-mêmes les «barbares". L'idée répandue - à tort dans nombre de cas - selon laquelle les Soviétiques ne faisaient pas de prisonniers renforça la tendance à l'œuvre. Mais ce sont pourtant les Allemands qui décidèrent d'une telle politique. On a donc affaire à un comportement mimétique par rapport à une image, et non par rapport à une « réalité » du comportement de l'ennemi.

14 L'apport de Bartov ne se limite toutefois pas à cette problématique. Il consacre d'intéressants commentaires critiques aux théories classiques sur la cohésion des groupes primaires au combat. D'après Bartov, la théorie en question est insuffisante car elle postule que le groupe doit être doté d'une certaine continuité (que l'on considère principalement acquise pendant l'entraînement). Or, une telle continuité n'existe pas dans de nombreuses situations de combat. Si le nombre d'hommes tués, ou blessés et évacués, est trop élevé, comme c'était le cas sur le front de l'Est, la stabilité du groupe devrait disparaitre. Et, par extension, les capacités de combat devraient être fortement altérées, ce qui n'a pas vraiment été prouvé. En d'autres termes, on peut se demander si l'attention ne doit pas davantage être portée sur la construction d'une identité de groupe plus générique, une cohésion institutionnelle et idéelle, que sur l'existence de groupes primaires considérés comme idéologiquement neutres ${ }^{12}$. 

de celui de Bartov ${ }^{13}$. Intitulé The American Samurai, l'ouvrage de Cameron, lui-même ancien fusilier de l'unité d'élite, est une étude sur la Première Division du corps des Marines entre 1941 et 1951. Elle se consacre majoritairement à la période qui s'étend de la campagne du Pacifique pendant la Seconde Guerre à la guerre de Corée. On remarquera au passage que l'auteur réalise des comparaisons peu flatteuses entre les Marines, issus d'une société démocratique, et la Wehrmacht à partir des travaux de Bartov.

Pour Cameron, le corps des Marines a provoqué la « brutalisation » du combat contre les Japonais au travers d'une identité institutionnelle de dureté et d'une image raciste de l'adversaire. Tout comme chez Bartov, une sorte de mimétisme est à l'œuvre. Les Marines se sont pris pour des samouraïs et ont reproduit les comportements qu'ils imputaient à l'Autre. Par ailleurs, Cameron insiste sur l'évolution des narrations identitaires. Loin d'être figées, et malgré leurs forces contraignantes, elles évoluent.

Résumons la thèse de Cameron. Tout d'abord, il insiste sur le fait que le corps des Marines apparaît au XIX ${ }^{\text {ème }}$ siècle comme une force coloniale et obscure. Les Marines sont l'outil de la gunboat diplomacy. On les considère au service du Département d'Etat et des grandes compagnies installées en Amérique du Sud. D'emblée, plusieurs cas de brutalités sévères sont évoqués vis-à-vis de leur comportement en Haïti, au Nicaragua ou en République Dominicaine à la fin du XIX ${ }^{\text {ème }}$ et au début du XX ${ }^{\mathrm{ème}}$ siècle. L'imagerie raciste est déjà enracinée au sein de l'unité.

18 Avec la Première Guerre mondiale et pendant l'entre-deux-guerres, le corps gagne en confiance et en renommée. Ses dirigeants exercent d'ailleurs avec doigté l'art des public relations. C'est aussi lors des années 1930 que les Marines se dotent d'une identité fonctionnelle, fondée sur les opérations amphibies, identité qui renforce leur position par rapport à l'US Navy et à l'US Army. Parallèlement, une identité propre est inculquée aux recrues. La dure sélection à l'entrée renforce déjà l'image d'élitisme. Ensuite, le Marine se voit séparé du monde féminin et civil ${ }^{14}$. Une éthique guerrière, " anti-civile ", misogyne, " homophobe » (en apparence à tout le moins) et raciste (les Marines sont parmi les plus réfractaires à l'engagement d'Afro-Américains) lui est dispensée. Il s'agit là d'une identité collective qui permet selon Cameron, comme chez Bartov, d'expliquer la cohésion des unités malgré les pertes élevées dans certaines situations.

19 Au début de la guerre, les Marines se sont donc forgé une image du Japonais répondant aux représentations racistes et populaires. Il est présenté comme un superman cruel et traitre. Ces représentations s'intègrent dans un schéma de négation de l'homme de couleur, schéma légué par les opérations coloniales. A contrario, l'image des Chinois, alliés des Etats-Unis, connaît une adaptation plus "positive ", malgré la différence de peau. Cependant, l'image en question nourrit aussi une attitude paternaliste.

$\mathrm{Au}$ total, les représentations de l'ennemi vont jouer un rôle fondamental dans la «brutalisation» du combat. A titre d'exemple, les Marines convaincus de la nature traître des Japonais ne leur font pas confiance lorsque ces derniers tentent de se rendre (les Japonais avaient également subi une propagande leur indiquant que les Marines ne faisaient pas de prisonniers). Ensuite, les Américains apprennent à se comporter au combat, dans la jungle, comme le font leur ennemi. Par extension, l'image de l'adversaire commence à évoluer, ou à se fragmenter. Parmi les journalistes ou les officiers qui ne se retrouvent pas au front, le Japonais est toujours considéré comme 
une brute animale. Sur le terrain, au contraire, le discernement est de mise quant à l'évaluation des capacités techniques et tactiques de l'ennemi.

Cameron souligne aussi que l'identité de la première division, opérant une distinction entre son statut d'élite et celui des autres forces, causa des difficultés quant aux possibilités de coopération avec des unités de l'armée de terre. Il mentionne également l'appui qu'apporta l'idéologie technicienne au processus de «brutalisation $»^{15}$. Ce phénomène est particulièrement bien décrit au travers de l'emploi du lance-flammes. Enfin, Cameron apporte quelques éléments intéressants sur l'effacement de la limite entre réalité et irréalité chez les combattants. Gavés d'un imaginaire populaire, romantique et aventurier, certains hommes avaient peine à croire, lors de leurs premiers combats, qu'ils vivaient quelque chose de réel et non un « jeu ».

Bien que beaucoup plus intéressée par la problématique d'adaptation organisationnelle au combat, l'étude de Michael Doubler ajoute un éclairage supplémentaire, quasiment « en creux », aux études de Cameron et Bartov ${ }^{16}$. L'ouvrage de Doubler est consacré aux membres de l'US Army déployés sur le théâtre européen lors de la Seconde Guerre mondiale. Il constate également l'importance de la cohésion sociale au sein des groupes primaires au combat et donne d'excellentes informations sur les problèmes psychologiques des hommes ${ }^{17}$. Toutefois, l'intérêt de sa démonstration, au regard de ce qui a été indiqué auparavant, est de constater l'absence de «barbarisation » de la part des soldats de l'US Army. Plus encore, Doubler indique que le manque de rancœur dont les hommes faisaient preuve vis-à-vis de l'ennemi inquiéta le commandement. Ainsi, la plupart des GIs n'étaient pas convaincus qu'il fallait punir les populations allemandes mais simplement leurs responsables politiques après le conflit. Par ailleurs, l'auteur évoque des cas d'évitements volontaires lors de rencontres fortuites entre soldats américains et allemands. Certes, il ne faut pas en inférer que les soldats américains en Europe n'ont jamais commis d'exactions. Mais, de telles actions n'ont été que le produit de circonstances particulières et non systématiques.

En conclusion, Doubler présente les GIs comme désireux d'en finir au plus tôt avec la guerre. Pour ce faire, leur objectif consistait à détruire un maximum de forces allemandes au travers d'offensives se succédant rapidement ${ }^{18}$. L'auteur évoque d'ailleurs les difficultés rencontrées par les hommes lorsqu'il fallut mener des opérations défensives, auxquelles ils étaient peu habitués, dans les Ardennes en 1944.

Comme on peut le voir, ces études de cas apportent une série d'explications riches à la question de l'intensité de la violence. Les facteurs identitaires et liés à la construction des groupes sociaux y sont prépondérants ${ }^{19}$. A contrario, l'absence de représentations par trop polarisées aurait tendance à limiter l'ascension aux extrêmes.

\section{« Brutalisation » discursive - distanciation réelle}

Dans le processus de "brutalisation", même les discours populaires ont de l'importance, comme le montre Edmund P. Russell en analysant les représentations de l'Autre sous la forme d'insectes à anéantir ${ }^{20}$. Le point de départ de son analyse est la constatation qu'en 1944-1945, deux images représentant des sujets mi-hommes, miinsectes ont été publiées dans des périodiques aux Etats-Unis. La première, issue d'un journal de l'industrie chimique, vante les mérites d'un insecticide. Les insectes y sont symbolisés par un Allemand, un Japonais et un Italien. La seconde, qui provient d'une publication du corps des Marines, présente les mérites de la bombe A qui va écraser les 
« insectes japonais ». Russell voit ici l'expression d'un lien entre l'industrie chimique, qui produit les insecticides, et la métaphore de l'ennemi sous-homme représenté sous les traits de l'insecte. Il commence par montrer les connections qui existent, depuis le début du $X X^{\text {ème }}$ siècle, entre l'armée et l'industrie chimique (gaz de combat et production de zyklon B pour les camps d'extermination) et détaille ensuite l'émergence de ce discours déshumanisant au sein de la culture populaire, légitimant de cette manière des stratégies d'anéantissement.

Tout au long de ce tour d'horizon, nous avons été amenés à mettre en évidence des études historiques relatives au processus de déshumanisation en temps de guerre. Toutefois, plusieurs politologues ont également apporté une contribution à cette thématique. Mentionnons tout d'abord l'étude postmoderne de Michael De Landa qui porte sur la comparaison de l'armée avec une machine, machine qui se nourrirait, par agrégations successives, de nouvelles techniques. A la lecture de De Landa, il est difficile de ne pas percevoir le jour déshumanisé de cette machine semblant gagner en indépendance par rapport à l'homme ${ }^{21}$.

Indiquons également l'étude de Jennifer Milliken et David Sylvan sur le processus de distanciation humaine dans le contexte des bombardements américains en Asie du Sud$\mathrm{Est}^{22}$. Les auteurs expliquent comment les ordres écrits à Washington et signés de la main du Président - c'est-à-dire un discours écrit - ont des effets réels en terme de souffrance sur le terrain. Ces ordres parviennent à produire une représentation " molle », fondée sur des théories stratégiques ${ }^{23}$ des victimes. Les représentations sont donc, dans ce cas, au cœur du processus de distanciation.

Enfin, l'ouvrage de Richard Wyn Jones, largement inspiré par les idées de Adorno et Horkheimer, sur la pensée stratégique constitue une contribution importante au débat ${ }^{24}$. Selon Wyn Jones, le propre des discours stratégiques est de traiter l'individu non plus dans son humanité mais en tant qu'objet. L'apparition d'un tel discours serait consubstantiel à l'émergence de la modernité. Cette dernière, au travers de l'affirmation de la rationalité sur le mode dichotomique moyen-fin, désacralisa et réifia l'individu. Selon Wyn Jones, Hiroshima et Auschwitz trouveraient leurs origines dans de telles constructions intellectuelles.

Au regard des conflits contemporains, souvent très brutaux eux aussi, il ne fait guère de doute que les éléments mis en évidence par ces différents auteurs mériteraient d'être exploités. Ils appellent indéniablement à de nouvelles recherches relatives aux rôles des représentations au sein des institutions politico-militaires en charge des décisions stratégiques et sur les moyens humains de distanciation.

\section{Evaluer l'Autre}

De longue date déjà, les historiens militaires se sont intéressés à la pensée stratégique et à son évolution. Aujourd'hui encore, ils continuent à produire une réflexion de premier plan sur le sujet ${ }^{25}$. De plus, certains politologues se sont eux-mêmes lancés dans de telles recherches sur le mode historique ${ }^{26}$. Toutefois, divers auteurs à la tournure résolument critique sont parvenus à renouveler les approches traditionnelles par de nouveaux questionnements.

31 A cet égard, l'ouvrage Strategy and Ethnocentrism de Ken Booth, publié en 1979, constituait déjà un bon embryon ${ }^{27}$. L'auteur y reprochait aux études stratégiques leur 
orientation trop ethnocentrique, leur manque d'intérêt pour les facteurs culturels et régionaux et leur attention trop développée sur la question des moyens, au détriment de l'évaluation des intentions. Bref, l'analyse stratégique avait des difficultés à penser l'Autre dans sa complexité.

On retrouvait des considérations assez similaires dans l'étude de Robert B. Bathrust intitulée Intelligence and the Mirror - On Creating an Enemy (1993) ${ }^{28}$. Encore marquée par l'expérience de la Guerre Froide, l'analyse de Bathrust soulevait la question de savoir comment était construite l'image de l'adversaire soviétique par les analystes américains. L'approche y était résolument inspirée par l'anthropologie (Edward Hall, Margaret Mead). Selon l'auteur, toute pensée se trouve enserrée dans des "boîtes", délimitée par des œillères. L'origine de ces limitations repose entre autres sur le fait que les individus doivent se mettre d'accord sur ce qui constitue la réalité, au travers de processus de négociations. Cependant, dans le cas d'analyses sur le comportement soviétique, ces négociations se déroulaient, assez logiquement, au sein de l'appareil de sécurité, entre Américains uniquement et donc sans "véritables" boucles de rétroaction avec l'Autre. Et Bathrust de souligner ensuite certaines caractéristiques des méthodes d'analyse américaines. Il met principalement en évidence le culte de la méthode rationnelle, envisagée à court terme et de façon indépendante du contexte socio-historique général. Ainsi, du point de vue américain, la compréhension de l'adversaire ne requérait pas, par exemple, la connaissance de l'idéologie marxisteléniniste. Comme Booth, Bathrust soulève le problème de la projection des critères d'analyse d'Ego sur l'Autre. Par ailleurs, il souligne que la méthode réaliste - rationnelle employée par les analystes américains tendait à renforcer une narration paranoïaque, de type théorie du complot, au sujet des Soviétiques.

\section{Production du savoir stratégique et création de l'ennemi}

Dans un registre plus socio-historique, les études de Christopher Simpson et de Ron Robin apportent un complément d'informations à la question de la création des savoirs stratégiques ${ }^{29}$. Les deux auteurs brossent un tableau très précis de l'émergence de ce que l'on pourrait nommer une "pensée stratégique psychologique et sociologique ». L'optique est ici liée aux rapports entre pouvoir et connaissance.

Selon Christopher Simpson, la discipline des études en communication doit sa naissance à un investissement massif, quoique parfois indirect, de la part des organismes de sécurité aux Etats-Unis entre 1945 et $1960^{30}$. L'étude, très fouillée, met à jour les liens institutionnels ayant existé entre les réseaux de chercheurs, les fondations privées, les think tanks et le gouvernement. On y apprend par exemple que des revues telles que The Public Opinion Quarterly, The American Journal of Sociology ou The American Sociological Review sont nées grâce à des financements émanant du Pentagone, de la CIA et du Département d'Etat. Raison pour laquelle les premières livraisons de ces revues se sont consacrées à des questions en rapport avec le concept de guerre psychologique. Simpson éclaire particulièrement bien le sujet et suggère que les recherches visées ont contribué à renforcer le sentiment d'insécurité propre à la Guerre Froide (par exemple à propos de tests de lancers de tracts de propagande menés, à grande échelle, sur les populations américaines). De plus, la question de la 
responsabilité des intellectuels de la défense dans ce processus se retrouve en filigrane tout au long du livre.

L'ouvrage de Simpson est utilement complété par celui de Ron Robin sur l'émergence des approches behavioristes en sociologie et leurs liens avec la recherche dans le domaine de la sécurité ${ }^{31}$. Ici aussi, les rapports entre scientifiques, fondations privées, think tanks et gouvernement sont mis en évidence. Robin montre que les contributions des sociologues et politologues portent sur des thématiques aussi diverses que la cohésion sociale au sein des forces armées, la modernisation des sociétés comme bouclier contre le communisme ou la guerre psychologique. L'auteur explique comment le souci de scientificité américain, refusant toute position idéologique, a fini par devenir une idéologie, de type technocratique et conservatrice, visant à la manipulation des masses. Il défend l'idée selon laquelle les intellectuels américains étudiés ont constamment été en quête de l'arme psychologique ultime, moyen de contrôle social total, équivalent de l'arme atomique en moins meurtrier. Le corpus ainsi développé amène à concevoir la tâche de ces scientifiques sous le jour de la croisade anticommuniste, contribuant ainsi à voir l'ennemi partout. L'hypothèse de l'Etat garnison de Harold Lasswell prend une nouvelle « validité » dans ce contexte, bien que dans une optique plus «molle $»^{32}$. Par ailleurs, la lecture de Robin tend à confirmer l'hypothèse de Booth et Bathrust sur l'ethnocentrisme propre à la recherche et l'accent prépondérant mis sur l'évaluation des capacités au détriment des intentions de l'adversaire.

\section{Clôture de l'espace et identité}

Si la narration de Robin et Simpson reste encore assez classiquement historique, tel n'est plus totalement le cas du passionnant ouvrage de Paul N. Edwards relatif à l'utilisation métaphorique de l'ordinateur durant la Guerre Froide ${ }^{33}$. Sur le fond pourtant, la matière analysée par Edwards recoupe celle de Robin et Simpson. Néanmoins, la volonté clairement affichée est de pratiquer une "contre-narration historique " qui remet en cause les discours dominants. La thèse centrale est celle du rôle de la technologie dans la formation de modes de pensée, thèse appliquée aux liens existants entre les ordinateurs, principalement développés au sein de l'institution militaire, et l'émergence des sciences cognitives. En effet, le paradigme de l'ordinateur est devenu dominant au sein de disciplines aussi diverses que la psychologie, la linguistique ou les neurosciences mais aussi, et c'est là un intérêt non négligeable de l'analyse, au sein des représentations populaires, cinématographiques ou romancées, de science fiction. Edwards nous guide pas à pas dans cet univers, évoquant le rôle des premiers ordinateurs militaires (la machine de Turing qui servait à décrypter les codes Enigma), l'élaboration d'une ceinture de radars antimissiles autour des Etats-Unis, le développement des capteurs et ordinateurs censés protéger le Vietnam du Sud (la ligne MacNamara), l'émergence de l'intelligence artificielle et de la cybernétique et ses liens avec les laboratoires comportementaux pendant la Seconde Guerre mondiale, etc. Outre sa grande richesse en informations, l'ouvrage met en évidence le questionnement foucaldien classique sur la création d'un panoptique ${ }^{34}$. En d'autres termes, Edwards suggère que l'ensemble des techniques liées à la métaphore de l'ordinateur, dans le contexte de la Guerre Froide, peut être interprété comme une tentative à grande échelle (mais avortée) de constituer un « monde fermé » (closed world) où tout peut être 
contrôlé de manière centralisée et automatisée. Monde fermé, mais aussi monde de la peur de l'Autre, encouragée par la clôture du bloc soviétique. Nul doute que la thèse d'Edwards puisse servir de base de réflexion aujourd'hui face à certains développements techniques contemporains dans le monde de la sécurité (par exemple, les projets Total Information Awareness, National Missile Defense et la Revolution in Military Affairs).

En plusieurs points, les idées développées par Michael Shapiro ${ }^{35}$ se révèlent complémentaires à celles d'Edwards. Selon Shapiro, il existe au sein de nos sociétés un discours identitaire fondé sur des représentations spatiales. L'imaginaire géographique joue ici un rôle de premier plan. Or, ce dernier plonge ses racines dans un savoir partiellement militarisé au travers des liens existant entre architecture militaire (pour la construction des forteresses) et urbanisme. Bref, les représentations spatiales servent de fondement à l'établissement des catégories Même et Autre, interne et externe, cohésion et chaos. En conclusion, se référant à Levinas et Derrida, Shapiro milite pour une éthique de la rencontre avec l'Autre, éthique qui serait résistante à toute conceptualisation (aconceptual), offrant la possibilité de reconnaissance infinie ${ }^{36}$.

\section{Quand la stratégie justifie l'Etat}

Enfin, la question du lien entre pouvoir et pensée stratégique peut être étudiée dans le contexte de la problématique de la création étatique. Dans ce registre, il est à noter que de longue date déjà, divers travaux socio-historiques ont mis en évidence le rôle de la guerre dans la formation étatique ${ }^{37}$. Des études plus récentes ont précisé davantage ce processus. Pour faire face aux menaces externes, les entités sociopolitiques européennes ont créé des forces armées modernes. L'établissement de celles-ci requérait des levées, toujours plus importantes, d'impôts et de troupes. La nécessité de disposer d'outils de gestion s'affirma alors. En découla logiquement la formation d'administrations et le renforcement de l'autorité étatique. Le processus de modernisation des forces armées déborde ensuite de l'Europe pour atteindre la Russie, le Japon, la Chine, l'Egypte, etc. Ce faisant, le modèle étatique connaît une grande diffusion et provoque l'occidentalisation du monde ${ }^{38}$.

Toutefois, il est possible d'inverser le raisonnement en question. La question qui se pose alors est de savoir si, comme l'indique Bradley S. Klein, la pensée stratégique ne sert pas de discours hégémonique permettant la reproduction de l'ordre étatique, favorable à certains groupes sociaux, et non un ensemble de propositions techniques idéologiquement neutres ${ }^{39}$. Cette perspective se trouvera complétée par celle de l'historien Joël Cornette ${ }^{40}$. D'après ce dernier, le fondement de la fonction royale à l'époque de Louis XIV repose sur la guerre ; la guerre confère à la royauté sa légitimité interne. Raison pour laquelle on retrouve de si nombreuses représentations, historiographiques et statuaires par exemple, mettant en scène le roi dans un univers conflictuel. Le rôle symbolique des représentations de type militaire, et en rapport avec le fait de posséder certaines catégories d'armements, dans le processus de renforcement de la représentation étatique auprès des populations a également été mis en évidence par plusieurs politologues constructivistes ${ }^{41}$. Au sein des Relations Internationales, ces travaux autorisent une nouvelle remise en cause du paradigme réaliste, paradigme inférant trop rapidement que la menace, dans un univers anarchique, c'est l'Autre. 

et de la construction de la réalité offrent un contrepoint théorique aux courants dominants des études stratégiques et de sécurité. Le panorama dépeint aurait certes pu inclure bien d'autres approches. On peut ainsi songer aux connaissances que livrent les études utilisant les notions de culture stratégique, culture militaire, culture de guerre et, dans un registre plus proche de la peace research, culture de paix. Les études relatives à la mémoire collective de la guerre pourraient aussi être considérées sous cet angle. Par ailleurs, les recherches se concentrant sur le rôle des réseaux de socialisation, intra et «transbureaucratiques », dans le contexte du «nouvel institutionnalisme » appliqué aux milieux de la défense, comportent souvent des éléments de discussion réflexive de premier plan ${ }^{42}$.

41

Malgré tout, les éléments mis en évidence offrent déjà une série de pistes d'investigation. Ils donnent un aperçu des moyens historiquement mis en œuvre en vue d'élaborer l'altérité. Ces déconstructions intellectuelles ont pour avantage, et pour limite parfois aussi, de nous renvoyer à nous-mêmes car, en dernière instance, il y a toujours un ego au cœur de l'altérité.

Enfin, ces quelques pistes incitent à interroger, et réinterroger notre monde. A leur lueur, il est difficile de ne pas se poser la question du rôle des représentations dans un conflit comme celui du Congo où les membres d'une milice se sont fait appeler Ninja. Il est également difficile de ne pas se demander comment a pu être construit l'Autre au cours de la guerre en ex-Yougoslavie et quels liens peuvent exister entre ces constructions et les exactions commises sur le terrain. Ou encore, de se poser la question de l'effet des représentations visuelles et virtuelles informatisées dans la façon dont les troupes américaines ont mené la guerre en Irak.

$\mathrm{Au}$ total, le champ de la réflexion, largement ouvert, suggère, entre autres, pour le politologue en Relations Internationales, la réintégration d'espaces plus limités dans son étude, et l'intégration de ces considérations dans des schémas critiques et constructivistes théoriques qui lui sont propres.

\section{NOTES}

1. Sur ce débat, voir par exemple l'excellent article de Ayse Ceyhan, «Analyser la sécurité: Campbell, Dillon et les autres », Cultures \& Conflits, n³1-32, Paris, L'Harmattan, automne 1998, pp. 39-62.

2. Voir par exemple l'introduction de Ronnie D. Lipschutz (dir.), On Security, New York, Columbia University Press, 1998 ; ainsi que la position plus ancienne de Jean Barrea, "La sécurité, c'est l'autre'", in David C.P. (dir.), Les études stratégiques - approches et concepts, Montréal, Méridien FEDN - Centre Québécois de Relations Internationales, 1989, pp. 417-434. Voir aussi: Buzan B., Waever O. et Wilde J. de, Security - A New Framework for Analysis, Boulder et Londres, Lynne Rienner, 1998.

3. Voir à ce propos Weldes J., Laffey M., Gusterson H. et Duvall R., « Introduction: Constructing Insecurity " in Weldes J. et al., Cultures of Insecurity - States, Communities, and the Production of 
Danger, (avant propos par Marcus G.), Londres et Minneapolis, University of Minnesota Press, 1999, p. 13.

4. Sur la démarche constructiviste en général, voir par exemple Watzlawick P. (dir.), L'Invention de la réalité - Comment savons-nous ce que nous croyons savoir ?, (trad. de l'allemand), Paris, Seuil, 1988.

5. Comme chez Alexander Wendt dans son article essentiel , " Anarchy is what states make of it: the social construction of power politics », International Organization, 46, 2, été 1992, pp. 391-425.

6. Shils E.A. et Janowitz M., "Cohesion and Disintegration in the Wehrmacht in World War II ", Public Opinion Quarterly, 12, 2, été 1948, pp. 280-315 ; Marshall S.L.A., Men against Fire, The Problem of Battle Command in Future War, New York, Morrow, 1954 (1947). (Célèbre pour avoir affirmé que seuls $25 \%$ des soldats américains faisaient feu au contact de l'ennemi pendant la Seconde Guerre mondiale) ; Creveld M. van, Fighting Power - German and U.S. Army Performance, 1939-1945, Londres, Arms and Armour Press, 1983.

7. A ce propos, on lira par exemple Coutau-Bégarie H., «L'histoire militaire entre la pensée stratégique et la nouvelle histoire ", Stratégique, 28, 4, Paris, 1985, pp. 61-73.

8. Keegan J., The Face of Battle, New York, Viking Press, 1976.

9. L'entreprise ainsi lancée au sein de l'histoire militaire peut être partiellement rapprochée de celle de la "sécurité humaine " au sein des études de sécurité. Voir par exemple l'article de Booth K., " Security and emancipation », Review of International Studies, 17, Cambridge, Cambridge University Press, octobre 1991, pp. 313-326.

10. Pour un exemple récent, voir Fussell P., A la guerre. Psychologie et comportements pendant la Seconde Guerre mondiale, (trad. de l'anglais), Paris, Seuil, 2003.

11. Bartov O., Hitler's Army - Soldiers, Nazis, and War in the Third Reich, New York et Oxford, Oxford University Press, 1991. On retrouve déjà la notion de "barbarisation » appliquée aux sociétés européennes chez l'historien George Mosse. Mosse tente de montrer comment ce processus permet de mieux comprendre l'émergence des deux conflits mondiaux et du totalitarisme. Mosse G.L., De la Grande Guerre au totalitarisme. La brutalisation des sociétés européennes, (trad. de l'anglais), Paris, Hachette, 2003.

12. Sur le rôle de la cohésion de groupe par rapport à l'expression de la violence et sur la déshumanisation de l'Autre, voir aussi Browning C., Des hommes ordinaires - Le 101ème bataillon de réserve de la police allemande et la Solution finale en Pologne, (trad. de l'anglais, préf. de Pierre VidalNaquet), Paris, Les Belles Lettres, 1994 (1992) ; Ingrao C., "Culture de guerre, imaginaire nazi, violence génocide. Le cas des cadres du S.D.», Revue d'histoire moderne et contemporaine, 47, 2, Paris, avril-juin 2000, pp. 265-289.

13. Cameron C.M., The American Samurai - Myth and Imagination in the Conduct of Battle in the First Marine Division 1941-1951, Cambridge, Cambridge University Press, 1994. Voir également Dower J.W., War Without Mercy - Race and Power in the Pacific War, New York, Pantheon, 1987.

14. On retrouvera aussi d'importants éléments à ce propos chez Echevarria A.J., After Clausewitz. German Military Thinkers Before the Great War, Lawrence, Kansas University Press, 2000. L'auteur montre bien la crainte des militaires vis-à-vis de l'embourgeoisement des sociétés à la fin du XIXème siècle. Des campagnes de «propagandes » nationalistes furent menées en Allemagne en défense des valeurs viriles et militaires pour contrer le pacifisme. La création du scoutisme, par Baden-Powell, répondait aussi à cette logique.

15. Sur le rôle de l'imaginaire technique dans la violence militaire, on consultera aussi Sherry M.S., The Rise of American Airpower - The Creation of Armageddon, New Haven et Londres, Yale University Press, 1987.

16. Doubler M.D., Closing with the Enemy. How the GIs Fought the War in Europe, 1944-1945, Lawrence, University Press of Kansas, 1994.

17. Problèmes à peine reconnus au sein du corps des marines à l'époque. On ne s'en étonne guère à la lueur de la culture machiste et « stoïque " prévalant. 
18. On peut cependant se demander si les comportements des GIs en Europe n'étaient pas motivés par une certaine idéologie technique de l'efficacité militaire.

19. Comme, par exemple, le port de l'uniforme qui peut constituer un masque induisant certains comportements agressifs. A ce propos, voir par exemple Ben-Ari E., « Masks and Soldiering: The Israeli Army and the Palestinian Uprising ", Cultural Anthropology, 4, 4, Arlington, American Anthropological Association, novembre 1989, pp. 372-389.

20. Russell E.P., "'Speaking of Annihiliation': Mobilizing for War Against Human and Insects Enemies, 1914-1945 », Journal of American History, 82, mars 1996, pp. 1505-1529.

21. Landa M. De, War in the Age of Intelligent Machines, New York, Swerve, 1991.

22. Milliken J. et Sylvan D., « Soft Bodies, Hard Targets, and Chic Theories: US Bombing Policy in Indochina », Millenium: Journal of International Studies, 25, 3, 1996, London, LSE Publications, pp. 321-359.

23. Ajoutons que sur l'aspect «anesthésiant » des théories stratégiques nucléaires auprès des populations, on lira Paul Boyer, «From Activism to Apathy: The American People and Nuclear Weapons, 1963-1980", The Journal of American History, 70, 4, Bloomington, the Organization of American Historians, mars 1984, pp. 821-844. D'après l'auteur, le jargon scientifique des théoriciens servit de bouclier à un débat au sein de la société civile.

24. Wyn Jones R., Security, Strategy, and Critical Theory, Colorado, Lynne Rienner, 1999.

25. Voir par exemple: Gat A., A History of Military Thought from Enlightenment to the Cold War, Oxford, Oxford University Press, 2001 ; Coutau-Bégarie H., Traité de stratégie, Paris, Economica / ISC, 1999.

26. En guise d'exemple, voir: Steiner B.H., Bernard Brodie and the Foundations of American Nuclear Strategy, Lawrence, University Press of Kansas, 1991.

27. Booth K., Strategy and Ethnocentrism, Londres, Croom Helm, 1979.

28. Bathrust R.B., Intelligence in the Mirror - On Creating an Enemy, Londres, PRIO - Sage, 1993.

29. Voir aussi la livraison d'automne 1995 de la Radical History Review (numéro spécial consacré aux savoirs experts pendant la Guerre Froide). La consultation de la revue Social Studies of Science n'est pas non plus inutile à ce sujet. Cette dernière contient des études essentielles sur les liens entre création des savoirs scientifiques, bureaucraties, pouvoir dans le contexte de la Guerre Froide. A titre d'exemple, voir: Fortun M., Schweber S.S., «Scientists and the Legacy of World War II: The Case of Operations Research (OR)», Social Studies of Science, 23, 4, Londres, Sage Publications, novembre 1993, pp. 595-642. Enfin, sur la question de la construction de la menace et du rôle des discours de la sécurité, nous renvoyons également aux articles publiés dans la revue Cultures et Conflits. Voir par exemple, Bigo D., "Grands débats dans un petit monde ", Cultures \& Conflits, n¹9/20, Paris, L'Harmattan, automne-hiver 1995, pp. 7-48.

30. Simpson C., Science of Coercion. Communication Research \& Psychological Warfare 1945-1960, Oxford, Oxford University Press, 1994. Voir aussi Oren I., « Is Culture Independant of National Security? How America's National Security Concern Shaped 'Political Culture' Research», European Journal of International Relations, 6, 4, Londres, Sage Publications, décembre 2000, pp. 543-573.

31. Robin R., The Making of the Cold War Enemy. Culture and Politics in the Military-Intellectual Complex, Princeton, Princeton University Press, 2001.

32. Lasswell H.D., "The Garrison State ", The American Journal of Sociology, 46, 4, Chicago, the University of Chicago Press, janvier 1941, pp. 455-468.

33. Edwards P.N., Closed World. Computers and the Politics of Discourse in Cold War America, Cambridge et Londres, MIT Press, 1996. Dans un genre plus « classique », l'étude de Fred Kaplan (The Wizards of Armageddon, New York, Touchstone, 1983.) sur la fabrication de la stratégie nucléaire américaine, reste également un apport de premier plan.

34. Foucault M., Surveiller et punir - Naissance de la prison, Paris, Gallimard, 1975. Ou une machine de vision pour paraphraser Paul Virilio, La Machine de vision, Paris, Galilée, 1988. 
35. Shapiro M.J., Violent Cartographies. Mapping Cultures of War, Minneapolis, University of Minnesota Press, 1997.

36. On prolongera utilement cette réflexion par celle développée par Jef Huysmans, en partie sur base de la notion Ami-Ennemi chez Carl Schmitt. Huysmans J., «Security! What Do You Mean? From Concept to Thick Signifier », European Journal of International Relations, 4, 2, Londres, Sage Publications, juin 1998, pp. 226-255. Par ailleurs, l'idée de Bill McSweeney selon laquelle le passage du concept de "défense " à celui de « sécurité " impliquant une déterritorialisation a également une certaine résonance ici. McSweeney B., Security, Identity and Interests. A Sociology of International Relations, Cambridge, Cambridge University Press, 1999. Enfin, les considérations de la branche critique de la géopolitique apportent aussi une lumière vive aux liens sécurité/espace. Voir par exemple O Tuathail G., Critical Geopolitics - The Politics of Writing Global Space, Londres, Routledge, 1996.

37. Voir par exemple Tilly C., "Reflections on the History of European State-Making » in Tilly C. (dir.), The Formation of National State in Western Europe, Princeton, Princeton University Press, 1975, pp. 3-83.

38. Parker G., The Military Revolution - Military Innovation and the Rise of the West, 1500-1800, Cambridge, Cambridge University Press, 1989 ; Downing B.M., The Military Revolution and Political Change - Origins of Democracy and Autocracy in Early Modern Europe, Princeton, Princeton University Press, 1993 ; Ralston D.B., Importing the European Army - The Introduction of European Military Techniques and Institutions into the Extra-European World 1600-1914, Chicago et Londres, Chicago University Press, 1990.

39. Klein B.S., Strategic Studies and World Order - The Global Politics of Deterrence, Cambridge, Cambridge University Press, 1994.

40. Cornette J., Le roi de guerre - Essai sur la souveraineté dans la France du Grand Siècle, Paris, Payot, 2000 (1993).

41. Kinsella D. et Chima J.S., "Symbols of statehood: military industrialization and public discourse in India ", Review of International Studies, 27, Cambridge, Cambridge University Press, 2002, pp. 353-373 ; Eyre D.P. et Suchman M., «Status, Norms, and the Proliferation of Conventional Weapons: An Institutional Theory Approach », in Katzenstein P.J. (dir.), The Culture of National Security - Norms and Identity in World Politics, New York, Columbia University Press, 1996, pp. 79-113.

42. Voir Farrell T., «Figuring Out Fighting Organizations: The New Organisational Analysis In Strategic Studies », The Journal of Strategic Studies, 19, 1, Taylor \& Francis, mars 1996, pp. 122-135.

\section{RÉSUMÉS}

Qu'est-ce que les approches intéressées par le rôle des représentations et la création de la réalité sont en mesure d'apporter à l'étude de la stratégie et de la guerre ? C'est à cette question que l'article tente d'apporter un éclairage en mettant en évidence l'apport de quelques travaux contemporains. Ceux-ci développent deux grandes catégories de considérations en la matière, chacune liée à la déshumanisation et la construction de l'Autre dans le domaine stratégique. Il s'agit en outre de croiser les recherches émanant de l'histoire, de la sociologie et des études de relations internationales. 
What do approaches interested by representations and the creation of reality bring to the study of strategy and war? This article tries to give an account to the question, showing the interest of some contemporary studies. These studies develop two great lines of considerations related to the topic, both pertaining to the dehumanisation of the construction of the Other by strategy. The analysis also aims at criss-crossing contributions from either history, sociology or international relations studies.

INDEX

Mots-clés : Stratégie, savoir, représentations, relations internationales

\section{AUTEUR}

\section{CHRISTOPHE WASINSKI}

Christophe Wasinski est doctorant en science politique. Il est assistant aux Facultés universitaires Notre-Dame de la Paix à Namur et à l'Université Libre de Bruxelles (REPI), et membre du Réseau Multidisciplinaire en Etudes Stratégiques. 